\title{
AN UPDATED CHECKLIST OF BIRDS OF SARISKA TIGER RESERVE, RAJASTHAN, INDIA
}

\section{Aisha Sultana}

Aravalli Biodiversity Park, Biodiversity Parks Programme, Centre for Environmental Management of Degraded Ecosystems, University of Delhi, Delhi 110007, India aishasultana28@yahoo.com

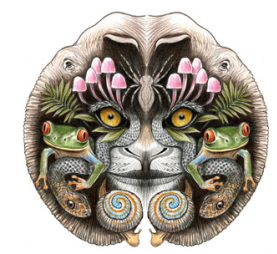

ISSN

Online 0974-7907 Print 0974-7893

OPEN ACCESS
Abstract: Surveys were carried out at 10 sites in the buffer and core zones of Sariska Tiger Reserve during 2007-2011. MacKinnon's species listing method was used to compile a checklist of birds. A total of 224 bird species was recorded including 36 new records. Ashy Drongo Dicrurus leucophaeus, Marshall's Iora Aegithina nigrolutea, Eurasian Eagle Owl Bubo bubo, Brown-headed Barbet Megalaima zeylanica, Indian Nightjar Caprimulgus asiaticus, Long-legged Buzzard Buteo rufinus, Northern Goshawk Accipiter gentilis, Red-necked Falcon Falco chicquera, Pheasant-tailed Jacana Hydrophasianus chirurgus, Red-whiskered Bulbul Pycnonotus jocosus, White-capped Water Redstart Chaimarrornis leucocephalus were some new records. Some important observations are given in detail.

Keywords: Abundance, Aravalli Hills, birds, disturbance, habitats, new records, Sariska Tiger Reserve, threatened.

Sariska Tiger Reserve lies $\left(27^{\circ} 05^{\prime}-27^{\circ} 33^{\prime} \mathrm{N} \& 79^{\circ} 17^{\prime}-\right.$ $76^{\circ} 34^{\prime} \mathrm{E}$ ) in the Alwar District of Rajasthan, India. The total area is $881 \mathrm{~km}^{2}$ with $497.8 \mathrm{~km}^{2}$ as its core area and consists of undulating plateaus and the wide valleys of the Aravallis (Kidwai et al. 2011) with tropical dry deciduous thorn forest (Champion \& Seth 1968). The topography of Sariska supports semi-deciduous riparian forest, scrub-thorn arid forests, dry deciduous forests, rocks and grasses (Shahabuddin et al. 2006). Anogeissus pendula is the dominant tree species of the forest whereas Boswellia serrata and Lannea coromandelica grow on rocky forested patches. Some valleys support Acacia catechu, Butea monosperma, Zizyphus mauritiana, Z. nummularia, Capparis separia and $C$. decidua. Besides these, some noteworthy tree species are Terminalia arjuna, Commiphora wightii, Sterculia urens, Emblica officinalis and Terminalia bellerica.

Sariska Tiger Reserve is rich in avifaunal diversity (Sankar et al. 1993; Shahabuddin et al. 2004, 2006). Owing to this high diversity, Sariska has been considered as an Important Bird Area (IBA code: IN-RJ-18) as recognised by Islam \& Rahmani (2004). Therefore, it is essential to survey this area for the documentation of avian fauna. A total of 10 sites was selected for a bird survey in the core and buffer zones of the reserve, i.e., Sariska, Silised Lake, Kanakwari, Karanakawas, Indradhauk Village, Kalighati, Pandupole, Bara, Kushalgarh and Kiraska (Fig. 1).

\section{Methods}

MacKinnon's species listing method (MacKinnon \& Philips 1993; Bibby et al. 2000) was adopted for the bird survey in Sariska Tiger Reserve in all seasons during 2007-2011. Bird lists were compiled in each area and each list comprised only 20 different bird species seen. No species were included more than once in the list.

DOI: http://dx.doi.org/10.11609/JoTT.o3215.4791-804 | ZooBank: urn:Isid:zoobank.org:pub:FD44AD9B-E3DE-4CC5-8D10-F75A18E4B020

Editor: R. Jayapal, SACON, Coimbatore, India.

Date of publication: 26 September 2013 (online \& print)

Manuscript details: Ms \# 03215 | Received 21 May 2012 | Final received 07 July 2013 | Finally accepted 28 August 2013

Citation: Sultana, A. (2013). An updated checklist of birds of Sariska Tiger Reserve, Rajasthan, India. Journal of Threatened Taxa 5(13): 4791-4804; http://dx.doi. org/10.11609/JoTT.03215.4791-804

Copyright: (C) Sultana 2013. Creative Commons Attribution 3.0 Unported License. JoTT allows unrestricted use of this article in any medium, reproduction and distribution by providing adequate credit to the authors and the source of publication.

Funding: Delhi Development Authority, Government of India (Project - Biodiversity Parks Programme)

Competing Interest: Authors declare no competing interest.

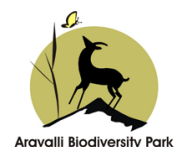

Acknowledgements: I am thankful to forest officials of Sariska Tiger Reserve for giving permission to survey in the area. Dr. Stephen is acknowledged for his help in the field. Thanks to anonymous reviewers for their fruitful comments and suggestions. 


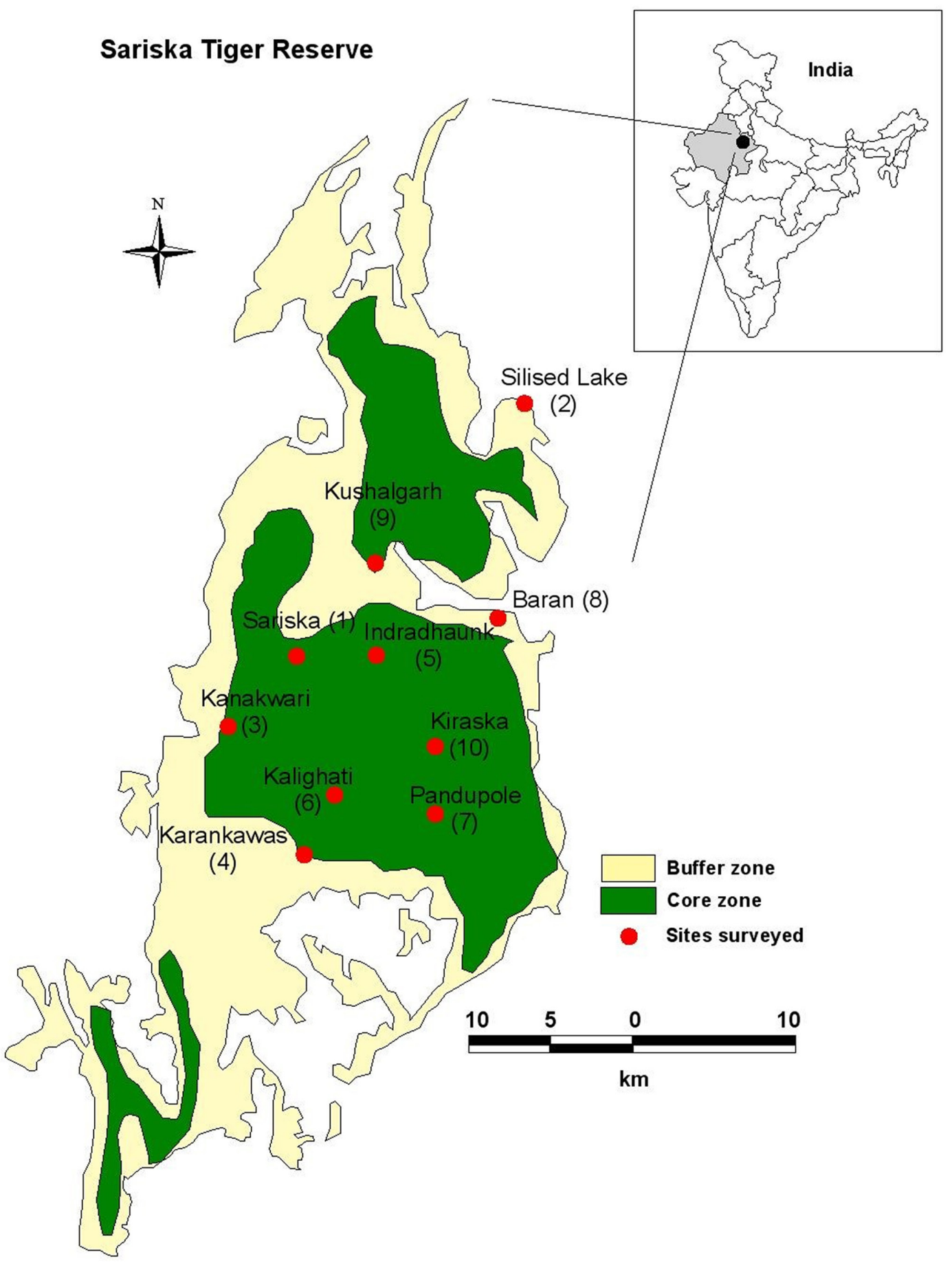

Figure 1. Map of Sariska Tiger Reserve, Rajasthan, India showing surveyed sites.

All lists were cumulated and a complete list of all bird species was prepared for each area. Different calls of confirmed species were also noted. The total number of each species from each site was added up and a checklist of birds for the tiger reserve compiled. All individual sightings of bird species were pooled and were ranked according to the following abundance categories: Stray
(S) (1 or 2 sightings), Occasional (0) (3-5 sightings), Uncommon (U) (6-15 sightings), Common (C) (16-50 sightings), Abundant (A) (> 50 sightings).

Earlier published lists of Sariska Tiger Reserve (Sankar et al. 1993; Shahabuddin et al. 2006) were considered for comparison. Different habitat types were also recognised for each species (where the species was 
sighted): $A=$ Aerial, $D=$ Disturbed, $F=$ Forested habitat, $\mathrm{G}=$ Grass dominant habitat, $\mathrm{O}=$ Open or scrub habitat, $\mathrm{W}=$ Water bodies and riparian habitat.

Feeding observations were also recorded in the field for each bird. The identification of birds and checklists were prepared following Grimmett et al. 1998.

\section{Results}

The appendix provides a complete list of birds ( 273 bird species of 53 families) that are known to occur in Sariska Tiger Reserve and this includes all previous records as well. In the present survey, a total of 224 bird species was recorded from different sites and habitats. Of these, 15 bird species were frugivorous, 32 omnivorous, 27 piscivorous, 114 insectivorous, 15 granivorous and 30 carnivorous in their feeding habits.

As compared with previously published lists, 36 new species of birds, including 57 species over and above Sanker et al. (1993) and 59 over and above Shahabuddin et al. (2006) were added to Sariska's avifauna in the present study. Some of the additions are: Pheasanttailed Jacana Hydrophasianus chirurgus, Plain leaf Warbler Phylloscopus neglectus, White-capped Water Redstart Chaimarrornis leucocephalus, Blue Rock Thrush Monticola solitarius, Rufous-fronted Prinia Prinia buchanani, Blue-cheeked Bee-eater Merops persicus, Grey-winged Blackbird Turdus boulboul, Long-legged Buzzard Buteo rufinus, Marshall's Iora, Tawny Eagle Aquila rapax, Red-whiskered Bulbul, Tawny Pipit Anthus campestris, Orange-headed Thrush Zoothera citrina, Northern Goshawk Accipiter gentilis, Red-necked Falcon Falco chicquera and Ultramarine Flycatcher Ficedula superciliaris. While some species which were recorded in the past were not recorded in the present survey such as the Blue-breasted Quail Coturnix chinensis, Greater Grey-headed Fish Eagle Ichthyophaga ichthyaetus, Eurasian Cuckoo Cuculus canorus, Tickell's Thrush Turdus unicolor, Common Iora Aegithina tiphia, Sarus Crane Grus antigone and Greater Flamingo Phoenicopterus ruber. A few species were considered to be commonly present in the area (Sankar et al. 1993; Shahabuddin et al. 2006) but could not be recorded during the present survey (see Appendix 1).

The status was recorded only for the birds recorded in the present study and it is clearly evident from Fig. 2 that residents (128 species) outnumber the winter (89) and summer visitors (7).

\section{Some notable observations}

White-capped Water Redstart Chaimarrornis leucocephalus: One bird was observed and photographed

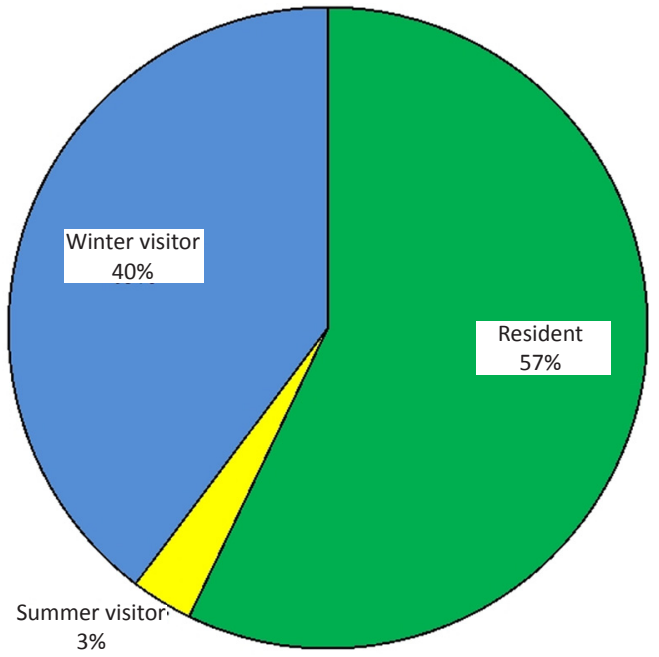

Figure 2. Status of the birds of Sariska Tiger Reserve surveyed during 2007-2011.

in Pandupole near a stream on 25 March 2007 and 26 March 2008. There are no other published records of this bird from this area. This bird is not known to migrate in Sariska Tiger Reserve so sighting was interesting although it was sighted wintering in Delhi (Sharma 2002) and in Bikaner District along the Indira Gandhi canal in Rajasthan (Kumar 2004).

Grey-winged Blackbird Turdus boulboul: This bird was recorded in Karanakawas (25 March 2007) and Pandupole (25 November 2009). Only two male individuals were seen once at each site. It is a new record for the area. It was observed feeding and searching the litter on the forest floor.

Marshall's Iora Aegithina nigrolutea: Four individuals; three male and one female were recorded from Kanakwari (24 March 2007) and Kalighati valley (25 November 2009). This species was not recorded by Sankar et al. 1993 and Shahabuddin et al. 2006 while another species Common Iora Aegithina tiphia, which was not recorded in the present survey, was recorded as rare by Sankar et al. 1993.

Northern Goshawk Accipiter gentilis: Two male individuals were sighted in the Kalighati valley on 26 December 2009 and 10 December 2011. One was observed feeding on a squirrel while another was perched in the thick foliage of a Holoptelea integrifolia tree. Both individuals had a black head and a diagnostic broad white supercilium. Underparts were grayishwhite and the eyes of one individual were orangish-red (the other's eyes could not be seen).

Eurasian Eagle Owl Bubo bubo: This bird was sighted four times in Kanakwari (8 August 2007) and Pandupole 
(18 November 2008, 30 April 2009 \& 11 December 2011) in the rocky forested area. One male was recorded calling in Kanakwari and three other males were sighted from the rocky hills of Pandupole area. Although no nest was sighted it seemed that it bred here.

Red-necked Falcon Falco chicquera: It was recorded perching on a Mallotus phillipanensis tree once in the Pandupole area (20 September 2010) in forest with open habitat. Given the species' declining range and populations in the subcontinent, the sighting of the Falcon in Sariska is particularly notable.

White-rumped Vulture Gyps bengalensis: This vulture was seen soaring in the Pandupole area near rocky cliffs twice only during 2008 on 10 June and 18 November in the same area. It was recorded as frequent by Sankar et al. 1993 and Shahabuddin et al. 2006 but it has since become very uncommon.

Long-billed Vulture Gyps indicus: In the present survey it was seen once in the Pandupole area on 21 September 2010. It was recorded as rare by Sankar et al. (1993), Shahabuddin et al. (2006) observed that the species occurred in sizeable numbers. However, no breeding signs were observed.

Red-headed Vulture Sarcogyps calvus: Uncommon in reserve and it was seen twice in the Khushalgarh area (8 August 2007 \& 11 June 2008) in the open habitat. No breeding signs were recorded.

Tawny Eagle Aquila rapax: A mature male was seen once near Karnakawas on 11 December 2011. It was a first record for the tiger reserve. Surprising that it was not recorded before, as Sariska has an ideal habitat in the species' core geographical range.

Long-legged Buzzard Buteo rufinus: This migratory buzzard was recorded once in Pandupole in 2010 on 20 September. It was seen feeding on a Rock Pigeon. The plumage was pale, eyes were dark and upperparts were rufous. No band was seen on the tail and seemed to be an adult.

Forest Wagtail Dendronanthus indicus: A confirmed sighting of this wagtail occurred on 8 March 2009 and 21 September 2010 at Silised Lake and Bara ki nadi, respectively. Four individuals were recorded at both sites. It was sighted in the nearby forest habitat of the Silised Lake and Bara ki nadi only and once it was recorded feeding with Citrine Wagtail and White Wagtail near water. This must be a passage migrant in Sariska.

Red-whiskered Bulbul Pycnonotus jocosus: This bird was sighted on 10 December 2011 in Silised Lake and Indradhaunk Village. A total of ten individuals was recorded. Indradhaunk Village is a degraded habitat and this bird was seen feeding on Lantana camara and
Ehretia laevis berries. It was never recorded from this area in the past (Sankar et al. 1993; Shahabuddin et al. 2006).

White-bellied Minivet Pericrocotus erythropygius: This is a resident bird species but uncommon in Sariska Tiger Reserve. It was recorded from Sariska (10 June 2008), Karankawas (20 September 2010), Pandupole (25 November 2009) and Kalighati areas (20 September 2010)

Ultramarine Flycatcher Ficedula superciliaris: It was recorded from Kanakwari (23 February 2007), Kalighati (10 December 2011), Pandupole (23 December 2009) and Khushalgarh (23 February 2007). One-two individuals were recorded from each of these areas. A white supercilium was very clear in the males, indicating the West Himalayan population of the nominate race. It was also sighted at the Bara Fort in Alwar (not a part of the Tiger Reserve) on 19 September 2010.

Orange-headed Thrush Zoothera citrina: This thrush was recorded occasionally from Pandupole (20 March 2010) and Kushalgarh (26 March 2008) area. But it is not reported in earlier studies (Sankar et al. 1993; Shahabuddin et al. 2006).

\section{Discussion}

Sariska Tiger Reserve is a significant conservation unit for the avifauna in the whole Aravalli Hills stretch. Many threatened bird (IUCN 2011) species were sighted here such as the Egyptian Vulture (Endangered), Whiterumped Vulture, Long-billed Vulture and Red-headed Vulture (Critically Endangered) and Laggar Falcon, Darter and Painted Stork (Near Threatened). The topography and diversity of habitats - for instance the water bodies, lakes, dense forest, scrub forest, open land and surrounded by agricultural fields provided different strata and guilds, which increased the diversity of birds. The presence of 22 raptor species including scavengers like the vulture signifies the importance of the area in terms of healthy ecosystems.

Out of 10 surveyed sites, the Kalighati Valley, Karankawas, Pandupole and Silised Lake were the most bird-rich areas. These areas can be considered highly conserved core blocks for birds. Sankar et al. (2010) also suggested the same areas for the conservation of the tiger. Many threatened bird species have been sighted in these areas only (Appendix 1). Silised Lake and its surrounding forest are very important for water birds including waders. Many passage migratory and migratory bird species were recorded here but this lake is under serious threat from tourism and local pressure. People from the surrounding villages use this lake for 
their daily needs. They collect fuel wood, fodder and timber from the nearby forest. Livestock grazing on the shore of the lake is another threat to the birds.

After the relocation of tigers, the habitat situation has been improved in Sariska but many threats still exist (Sankar et al. 2010). It has already been reported that biomass extraction is leading to the changes in vegetation composition of the reserve (Kumar \& Shahabuddin 2005; Gupta \& Yadav 2005; Yadav \& Gupta 2006, 2007) and it is changing the bird composition also. Many new bird species were recorded only from a specific area, which is under threat by tourism. Pandupole Temple attracts thousands of religious tourists during June-September (Ministry of Environment and Forests 2006) and this poses a potential threat to the mesic ecosystem of the area which is known for its high galliform diversity and density (Kidwai et al. 2011). Further surveys are still required in the buffer zone of the tiger reserve for vulture population assessment as they were not recorded from these areas in the present survey.

\section{REFERENCES}

Bibby, C.J., N.D. Burgess, D.A. Hill \& S.H. Mustoe (2000). Bird Census Techniques - Second Edition. Academic Press, London.

Champion, H.G. \& S.K. Seth (1968). A Revised Survey of the Forest Types of India. Government of India Publication, Delhi.

Grimmett, R., C. Inskipp \& T. Inskipp (1998). Birds of the Indian Subcontinent. Oxford University Press, Oxford, U.K.

Gupta, S.K. \& A.S. Yadav (2005). Population structure of tress species in the Sariska Tiger Reserve: Effects of aspects of hill slopes and human disturbance. Bulletin of the National Institute of Ecology 15: 35-41.

Islam, M.Z. \& A. Rahmani (2004). Important Bird Areas in India: Priority Sites for Conservation. Indian Bird Conservation Network: Bombay Natural History Society and Birdlife International (UK), Mumbai.

IUCN (2011). IUCN Red List of Threatened Species. Version 2011.2.

Kidwai, Z., K. Sankar, Q. Qureshi \& J.A. Khan (2011). Abundance and habitat utilization by Galliformes in the Sariska Tiger Reserve, Rajasthan, India. International Journal of Galliformes Conservation 2: 54-60.

Kumar, R. \& G. Shahabuddin (2005). Effects of biomass extraction on vegetation structure, density and composition of Indian tropical dry forest. Environmental Conservation 32(2): 1-12.

MacKinnon, S. \& K. Phillips (1993). A Field Guide to The Birds of Borneo, Sumatra, Java and Bali. Oxford University Press, Oxford, U.K.

Ministry of Environment and Forests (2006). Evaluation reports of Tiger Reserves in India. Project Tiger Directorate, Government of India, India.

Sankar, K., D. Mohan \& S. Pandey (1993). Birds of Sariska Tiger Reserve, Rajasthan, India. Forktail 8: 133-141.

Sankar, K., Q. Qureshi, P. Nigam, P. K. Malik, P. R. Sinha, R. N. Mehrotra, R. Gopal, S. Bhattacharjee, K. Mondal \& S. Gupta (2010). Monitoring of reintroduced tigers in Sariska Tiger Reserve, western India: preliminary findings on home range, prey selection and food habits. Tropical Conservation Science 3(3): 301-318.

Shahabuddin, G., R. Kumar \& A. Verma (2006). Annotated checklist of the birds of Sariska Tiger Reserve, Rajasthan, India. Indian Birds 2(3): 71-76.

Shahabuddin, G., A. Verma \& R. Kumar (2004). Birds, forests and conservation: Critical issues in Sariska Tiger reserve, Rajasthan, India. Newsletter for Ornithologists 1(6): 82-84.

Yadav, A.S. \& S.K. Gupta (2006). Effect of micro-environment and human disturbance on the diversity of woody species in Sariska Tiger Project in India. Forest Ecology and Management 225: 178189; http://dx.doi.org/10.1016/j.foreco.2005.12.058

Yadav, A.S. \& S.K. Gupta (2007). Effect of micro-environment and human disturbance on the diversity of herbaceous species in Sariska Tiger Project. Tropical Ecology 48(1): 125-128. 
Appendix 1. Birds recorded from Sariska Tiger Reserve, India

\begin{tabular}{|c|c|c|c|c|c|c|c|}
\hline & Family / Species & Habitat & *Locality & Abundance & $\begin{array}{l}\text { Sankar } \\
\text { et al. (1993) }\end{array}$ & $\begin{array}{l}\text { Shahabuddin } \\
\text { et al. (2006) }\end{array}$ & $\begin{array}{l}\text { Threatened } \\
\text { status }\end{array}$ \\
\hline & Phasianidae & & & & & & \\
\hline 1 & Black Francolin Francolinus francolinus & $\mathrm{F}$ & $4,7,9$ & $\mathrm{O}$ & 1 & 1 & LC \\
\hline 2 & Grey Francolin Francolinus pondicerianus & $\mathrm{D}, \mathrm{O}$ & $1,2,3,4,5,7,9,10$ & A & 1 & 1 & LC \\
\hline 3 & Rain Quail Coturnix coromandelica & $\mathrm{D}, \mathrm{O}$ & 4,7 & 0 & 1 & 1 & LC \\
\hline 4 & Blue-breasted Quail Coturnix chinensis & - & - & - & 1 & - & LC \\
\hline 5 & Jungle Bush Quail Perdicula asiatica & $\mathrm{F}, \mathrm{O}$ & 7,9 & U & 1 & 1 & LC \\
\hline 6 & Red Spurfowl Galloperdix spadicea & $D, F$ & 7 & $\mathrm{~S}$ & 1 & - & LC \\
\hline 7 & Painted Spurfowl Galloperdix lunulata & $\mathrm{F}$ & 7 & 0 & 1 & 1 & LC \\
\hline \multirow[t]{2}{*}{8} & Indian Peafowl Pavo cristatus & $D, F, G, O$ & $1,3,4,5,6,7,8,9,10$ & A & 1 & 1 & LC \\
\hline & Anatidae & & & & & & \\
\hline 9 & Greylag Goose Anser anser & W & 2 & $u$ & - & 1 & LC \\
\hline 10 & Bar-headed Goose Anser indicus & W & 2 & $u$ & - & 1 & LC \\
\hline 11 & Ruddy Shelduck Tadorna ferruginea & w & 2 & C & - & 1 & LC \\
\hline 12 & Comb Duck Sarkidiornis melanotos & - & - & - & 1 & - & LC \\
\hline 13 & Cotton Pygmy-goose Nettapus coromandelianus & - & - & - & 1 & - & LC \\
\hline 14 & Gadwall Anas strepera & W & 2 & C & 1 & 1 & LC \\
\hline 15 & Eurasian Wigeon Anas penelope & W & 2 & $u$ & - & - & LC \\
\hline 16 & Mallard Anas platyrhynchos & W & 2 & C & 1 & 1 & LC \\
\hline 17 & Spot-billed Duck Anas poecilorhyncha & W & $1,2,8$ & c & 1 & 1 & LC \\
\hline 18 & Northern Shoveler Anas clypeata & W & 2 & $u$ & 1 & 1 & LC \\
\hline 19 & Northern Pintail Anas acuta & W & 2 & C & 1 & 1 & LC \\
\hline 20 & Garganey Anas querquedula & W & 2 & U & - & 1 & LC \\
\hline 21 & Common Teal Anas crecca & W & 2 & $u$ & 1 & 1 & LC \\
\hline 22 & Red-crested Pochard Rhodonessa rufina & W & 2 & $u$ & 1 & - & LC \\
\hline 23 & Common Pochard Aythya ferina & - & - & - & 1 & 1 & LC \\
\hline \multirow[t]{2}{*}{24} & Tufted Duck Aythya fuligula & - & - & - & 1 & - & LC \\
\hline & Turnicidae & & & & & & \\
\hline \multirow[t]{2}{*}{25} & Small Buttonquail Turnix sylvatica & $D, F$ & $1,3,4,6,7$ & $u$ & 1 & - & LC \\
\hline & Picidae & & & & & & \\
\hline 26 & Eurasian Wryneck Jynx torquilla & $D, F, O$ & $1,3,4,5,6,9$ & C & - & 1 & LC \\
\hline 27 & $\begin{array}{l}\text { Brown-capped Pygmy Woodpecker } \\
\text { Dendrocopos nanus }\end{array}$ & - & - & - & 1 & 1 & LC \\
\hline 28 & $\begin{array}{l}\text { Black-rumped Flameback Dinopium } \\
\text { benghalense }\end{array}$ & $\mathrm{F}, \mathrm{O}$ & $1,2,3,4,5,6,9,10$ & C & 1 & 1 & LC \\
\hline \multirow[t]{2}{*}{29} & $\begin{array}{l}\text { Yellow-crowned Woodpecker Dendrocopos } \\
\text { mahrattensis }\end{array}$ & $\mathrm{D}, \mathrm{F}, \mathrm{O}$ & $1,3,5,6,7,9,10$ & C & 1 & 1 & LC \\
\hline & Capitonidae & & & & & & \\
\hline 30 & Brown-headed Barbet Megalaima zeylanica & $\mathrm{D}, \mathrm{F}, \mathrm{O}$ & $2,4,5,7,8,9$ & C & - & - & LC \\
\hline 31 & Coppersmith Barbet Megalaima haemacephala & $\mathrm{D}, \mathrm{F}, \mathrm{O}$ & $1,2,3,4,5,6,7,9,10$ & C & 1 & 1 & LC \\
\hline
\end{tabular}




\begin{tabular}{|c|c|c|c|c|c|c|c|}
\hline & Family / Species & Habitat & *Locality & Abundance & $\begin{array}{c}\text { Sankar } \\
\text { et al. (1993) }\end{array}$ & $\begin{array}{l}\text { Shahabuddin } \\
\text { et al. (2006) }\end{array}$ & $\begin{array}{c}\text { Threatened } \\
\text { status }\end{array}$ \\
\hline & Bucerotidae & & & & & & \\
\hline \multirow[t]{2}{*}{32} & Indian Grey Hornbill Ocyceros birostris & $D, F$ & $2,5,7$ & $S$ & 1 & - & LC \\
\hline & Upupidae & & & & & & \\
\hline \multirow[t]{2}{*}{33} & Common Hoopoe Upupa epops & $\mathrm{D}, \mathrm{O}$ & $1,3,4,5,7$ & C & 1 & 1 & LC \\
\hline & Coraciidae & & & & & & \\
\hline \multirow[t]{2}{*}{34} & Indian Roller Coracias benghalensis & $\mathrm{D}, \mathrm{O}$ & $2,3,4,5,7,9$ & $u$ & 1 & 1 & LC \\
\hline & Alcedinidae & & & & & & \\
\hline \multirow[t]{2}{*}{35} & Common Kingfisher Alcedo atthis & W & $2,4,6,8$ & C & 1 & 1 & LC \\
\hline & Halcyonidae & & & & & & \\
\hline \multirow[t]{2}{*}{36} & White-throated Kingfisher Halcyon smyrnensis & $D, F, O, W$ & $2,5,6,8,9,10$ & C & 1 & 1 & LC \\
\hline & Cerylidae & & & & & & \\
\hline \multirow[t]{2}{*}{37} & Pied Kingfisher Ceryle rudis & W & $2,8,10$ & C & 1 & 1 & LC \\
\hline & Meropidae & & & & & & \\
\hline 38 & Green Bee-eater Merops orientalis & $D, G, O$ & $1,2,3,4,5,6,7,8,9,10$ & C & 1 & 1 & LC \\
\hline 39 & Blue-cheeked Bee-eater Merops persicus & $D, W$ & $2,6,8$ & S & - & - & LC \\
\hline \multirow[t]{2}{*}{40} & Blue-tailed Bee-eater Merops philippinus & $\mathrm{D}, \mathrm{F}, \mathrm{O}$ & $1,3,4,5,6,7,9,10$ & $\mathrm{C}$ & 1 & 1 & LC \\
\hline & Cuculidae & & & & & & \\
\hline 41 & Pied Cuckoo Clamator jacobinus & $D, F$ & $1,4,6,7,9$ & C & 1 & 1 & LC \\
\hline 42 & Common Hawk Cuckoo Hierococcyx varius & $\mathrm{F}$ & $1,3,4,6,7$ & $u$ & 1 & 1 & LC \\
\hline 43 & Indian Cuckoo Cuculus micropterus & - & - & - & 1 & - & LC \\
\hline 44 & Eurasian Cuckoo Cuculus canorus & - & - & - & - & 1 & LC \\
\hline 45 & Asian Koel Eudynamys scolopacea & $\mathrm{D}, \mathrm{F}, \mathrm{O}$ & $1,2,3,4,5,6,7,9$ & C & 1 & 1 & LC \\
\hline 46 & Sirkeer Malkoha Phaenicophaeus leschenaultii & $\mathrm{F}$ & $3,6,7$ & $u$ & 1 & - & LC \\
\hline \multirow[t]{2}{*}{47} & Greater Coucal Centropus sinensis & $\mathrm{D}, \mathrm{O}$ & $1,3,4,6,7$ & C & 1 & 1 & LC \\
\hline & Psittacidae & & & & & & \\
\hline 48 & Alexandrine Parakeet Psittacula eupatria & $\mathrm{D}, \mathrm{F}, \mathrm{O}$ & $1,2,3,4,6,7,9,10$ & C & 1 & - & LC \\
\hline 49 & Rose-ringed Parakeet Psittacula krameri & $A, D, F, O$ & $1,2,3,4,5,6,7,8,9,10$ & A & 1 & 1 & LC \\
\hline \multirow[t]{2}{*}{50} & Plum-headed Parakeet Psittacula cyanocephala & $A, D, F$ & $1,2,3,4,6,7,9,10$ & C & 1 & 1 & LC \\
\hline & Apodidae & & & & & & \\
\hline \multirow[t]{2}{*}{51} & House Swift Apus affinis & & & & 1 & 1 & LC \\
\hline & Tytonidae & & & & & & \\
\hline \multirow[t]{2}{*}{52} & Barn Owl Tyto alba & $\mathrm{F}$ & 3,6 & 0 & 1 & - & LC \\
\hline & Strigidae & & & & & & \\
\hline 53 & Collared Scops Owl Otus bakkamoena & $D, F$ & $1,6,7$ & 0 & 1 & 1 & LC \\
\hline 54 & Eurasian Eagle Owl Bubo bubo & $\mathrm{F}$ & 3,7 & $\mathrm{O}$ & - & - & LC \\
\hline 55 & Brown Fish Owl Ketupa zeylonensis & W & 8 & S & 1 & 1 & LC \\
\hline 56 & Mottled Wood Owl Strix ocellata & - & - & - & 1 & - & LC \\
\hline 57 & Spotted Owlet Athene brama & $D, F, O$ & $1,3,4,6,7,8,10$ & C & 1 & 1 & LC \\
\hline
\end{tabular}




\begin{tabular}{|c|c|c|c|c|c|c|c|}
\hline & Family / Species & Habitat & *Locality & Abundance & $\begin{array}{c}\text { Sankar } \\
\text { et al. (1993) }\end{array}$ & $\begin{array}{l}\text { Shahabuddin } \\
\text { et al. (2006) }\end{array}$ & $\begin{array}{l}\text { Threatened } \\
\text { status }\end{array}$ \\
\hline \multirow[t]{2}{*}{58} & Short-eared Owl Asio flammeus & $\mathrm{F}$ & 4,7 & $\mathrm{~S}$ & 1 & - & LC \\
\hline & Caprimulgidae & & & & & & \\
\hline 59 & Grey Nightjar Caprimulgus indicus & $\mathrm{F}$ & $1,3,4,7,9$ & 0 & 1 & 1 & LC \\
\hline \multirow[t]{2}{*}{60} & Indian Nightjar Caprimulgus asiaticus & $\mathrm{F}$ & 6 & S & - & - & LC \\
\hline & Columbidae & & & & & & \\
\hline 61 & Rock Pigeon Columba livia & $A, D, O$ & $1,2,3,4,5,6,7,8,9,10$ & A & 1 & 1 & LC \\
\hline 62 & Oriental Turtle Dove Streptopelia orientalis & - & - & - & 1 & - & LC \\
\hline 63 & Laughing Dove Streptopelia senegalensis & $A, D, O$ & $1,2,3,4,5,6,7,8,9,10$ & A & 1 & 1 & LC \\
\hline 64 & Spotted Dove Streptopelia chinensis & $\mathrm{D}, \mathrm{O}$ & $2,5,8$ & S & 1 & 1 & LC \\
\hline 65 & Red Collared Dove Streptopelia tranquebarica & $\mathrm{D}, \mathrm{O}$ & $6,7,9$ & $\mathrm{O}$ & 1 & 1 & LC \\
\hline 66 & Eurasian Collared Dove Streptopelia decaocto & $D, F, O$ & $1,3,4,5,7,9,10$ & C & 1 & 1 & LC \\
\hline \multirow[t]{2}{*}{67} & $\begin{array}{l}\text { Yellow-footed Green Pigeon Treron } \\
\text { phoenicoptera }\end{array}$ & $\mathrm{D}, \mathrm{F}, \mathrm{O}$ & $1,5,6,7,8,9$ & C & 1 & 1 & LC \\
\hline & Gruidae & & & & & & \\
\hline \multirow[t]{2}{*}{68} & Sarus Crane Grus antigone & & & & 1 & - & VU \\
\hline & Rallidae & & & & & & \\
\hline 69 & $\begin{array}{l}\text { White-breasted Waterhen Amaurornis } \\
\text { phoenicurus }\end{array}$ & W & $1,2,8$ & C & 1 & 1 & LC \\
\hline 70 & Purple Swamphen Porphyrio porphyrio & W & $1,2,4,8$ & $u$ & 1 & 1 & LC \\
\hline 71 & Common Moorhen Gallinula chloropus & W & $1,2,8$ & $u$ & 1 & 1 & LC \\
\hline \multirow[t]{2}{*}{72} & Common Coot Fulica atra & W & 1,2 & C & 1 & 1 & LC \\
\hline & Pteroclidae & & & & & & \\
\hline 73 & Chestnut-bellied Sandgrouse Pterocles exustus & $\mathrm{D}, \mathrm{G}, \mathrm{O}$ & $1,6,7$ & 0 & 1 & - & LC \\
\hline 74 & Black-bellied Sandgrouse Pterocles orientalis & - & - & - & 1 & - & LC \\
\hline \multirow[t]{2}{*}{75} & Painted Sandgrouse Pterocles indicus & $\mathrm{F}, \mathrm{O}$ & $1,4,7$ & $u$ & 1 & - & LC \\
\hline & Scolopacidae & & & & & & \\
\hline 76 & Common Snipe Gallinago gallinago & w & 1,8 & $u$ & - & - & LC \\
\hline 77 & Black-tailed Godwit Limosa limosa & W & 8 & 0 & 1 & 1 & LC \\
\hline 78 & Common Redshank Tringa totanus & W & $1,2,8$ & C & 1 & 1 & LC \\
\hline 79 & Common Greenshank Tringa nebularia & W & $1,2,8$ & C & 1 & 1 & LC \\
\hline 80 & Green Sandpiper Tringa ochropus & W & 2 & C & 1 & 1 & LC \\
\hline 81 & Wood Sandpiper Tringa glareola & w & 1,2 & C & 1 & 1 & LC \\
\hline 82 & Common Sandpiper Actitis hypoleucos & W & 1,2 & C & 1 & 1 & LC \\
\hline 83 & Little Stint Calidris minuta & w & 8 & C & 1 & 1 & LC \\
\hline \multirow[t]{2}{*}{84} & Temminck's Stint Calidris temminckii & - & - & - & 1 & - & LC \\
\hline & Jacanidae & & & & & & \\
\hline \multirow[t]{2}{*}{85} & $\begin{array}{l}\text { Pheasant-tailed Jacana Hydrophasianus } \\
\text { chirurgus }\end{array}$ & W & $1,2,4$ & 0 & - & - & LC \\
\hline & Burhinidae & & & & & & \\
\hline 86 & Eurasian Thick-knee Burhinus oedicnemus & $\mathrm{F}, \mathrm{O}$ & $3,6,7$ & $\mathrm{o}$ & 1 & 1 & LC \\
\hline
\end{tabular}




\begin{tabular}{|c|c|c|c|c|c|c|c|}
\hline & Family / Species & Habitat & *Locality & Abundance & $\begin{array}{c}\text { Sankar } \\
\text { et al. (1993) }\end{array}$ & $\begin{array}{l}\text { Shahabuddin } \\
\text { et al. (2006) }\end{array}$ & $\begin{array}{l}\text { Threatened } \\
\text { status }\end{array}$ \\
\hline & Charadriidae & & & & & & \\
\hline 87 & Black-winged Stilt Himantopus himantopus & W & $1,2,8$ & C & 1 & 1 & LC \\
\hline 88 & Pied Avocet Recurvirostra avosetta & W & 2 & 0 & 1 & - & LC \\
\hline 89 & Little Ringed Plover Charadrius dubius & W & $1,2,4$ & $u$ & 1 & 1 & LC \\
\hline 90 & Kentish Plover Charadrius alexandrinus & - & - & - & 1 & - & LC \\
\hline 91 & Northern Lapwing Vanellus vanellus & - & - & - & 1 & - & LC \\
\hline 92 & Yellow-wattled Lapwing Vanellus malarbaricus & $\mathrm{G}, \mathrm{O}$ & $1,3,4,7,9,10$ & $u$ & 1 & - & LC \\
\hline 93 & River Lapwing Vanellus duvaucelii & - & - & - & 1 & - & LC \\
\hline 94 & Red-wattled Lapwing Vanellus indicus & $\mathrm{O}$ & $1,3,5,6,10$ & C & 1 & 1 & LC \\
\hline \multirow[t]{2}{*}{95} & White-tailed Lapwing Vanellus leucurus & 0 & 4,6 & 0 & - & 1 & LC \\
\hline & Laridae & & & & & & \\
\hline 96 & Brown-headed Gull Larus brunnicephalus & - & - & - & 1 & - & LC \\
\hline 97 & Black-headed Gull Larus ridibundus & W & 2 & $\mathrm{~S}$ & - & - & LC \\
\hline 98 & River Tern Sterna aurantia & W & 2 & $u$ & 1 & 1 & LC \\
\hline 99 & Black-bellied Tern Sterna acuticauda & W & 2,4 & 0 & 1 & 1 & LC \\
\hline 100 & Whiskered Tern Chlidonias hybridus & W & 2 & S & 1 & 1 & LC \\
\hline \multirow[t]{2}{*}{101} & White-winged Tern Chlidonias leucopterus & - & - & - & - & 1 & LC \\
\hline & Accipitridae & & & & & & \\
\hline 102 & Osprey Pandion haliaetus & - & - & - & - & 1 & LC \\
\hline 103 & Oriental Honey-buzzard Pernis ptilorhyncus & $\mathrm{F}$ & 3,6 & 0 & 1 & 1 & LC \\
\hline 104 & Black-shouldered Kite Elanus caeruleus & $\mathrm{D}, \mathrm{F}, \mathrm{O}$ & $1,2,3,5,6,7,9,10$ & C & 1 & 1 & LC \\
\hline 105 & $\begin{array}{l}\text { Grey-headed Fish Eagle Ichthyophaga } \\
\text { ichthyaetus }\end{array}$ & - & - & - & - & 1 & NT \\
\hline 106 & Egyptian Vulture Neophron percnopterus & $\mathrm{A}, \mathrm{O}$ & 6,7 & $u$ & 1 & 1 & EN \\
\hline 107 & White-rumped Vulture Gyps bengalensis & 0 & 7 & S & 1 & 1 & CR \\
\hline 108 & Long-billed Vulture Gyps indicus & 0 & 7 & S & 1 & 1 & CR \\
\hline 109 & Eurasian Griffon Gyps fulvus & $\mathrm{D}, \mathrm{O}$ & $3,5,7,10$ & $u$ & 1 & 1 & LC \\
\hline 110 & Red-headed Vulture Sarcogyps calvus & $\mathrm{O}$ & 9 & S & 1 & 1 & CR \\
\hline 111 & Crested Serpent Eagle Spilornis cheela & $\mathrm{F}$ & 3,6 & 0 & 1 & 1 & LC \\
\hline 112 & Eurasian Marsh Harrier Circus aeruginosus & W & 2 & S & 1 & 1 & LC \\
\hline 113 & Shikra Accipiter badius & $\mathrm{F}$ & $1,4,5,6,7,9,10$ & C & 1 & 1 & LC \\
\hline 114 & Northern Goshawk Accipiter gentilis & $\mathrm{F}$ & 6 & $\mathrm{~S}$ & - & - & LC \\
\hline 115 & Eurasian Sparrowhawk Accipiter nisus & - & - & - & 1 & 1 & LC \\
\hline 116 & White-eyed Buzzard Butastur teesa & $\mathrm{F}$ & 4,6 & 0 & 1 & 1 & LC \\
\hline 117 & Long-legged Buzzard Buteo rufinus & $\mathrm{F}, \mathrm{O}$ & 7 & S & - & - & LC \\
\hline 118 & Greater Spotted Eagle Aquila clanga & - & - & - & 1 & - & VU \\
\hline 119 & Tawny Eagle Aquila rapax & $\mathrm{F}$ & 4 & S & - & - & LC \\
\hline 120 & Imperial Eagle Aquila heliaca & $\mathrm{F}$ & 3 & S & 1 & - & LC \\
\hline 121 & Bonelli's Eagle Hieraaetus fasciatus & $\mathrm{F}$ & 7 & 0 & - & 1 & LC \\
\hline
\end{tabular}




\begin{tabular}{|c|c|c|c|c|c|c|c|}
\hline & Family / Species & Habitat & *Locality & Abundance & $\begin{array}{c}\text { Sankar } \\
\text { et al. (1993) }\end{array}$ & $\begin{array}{l}\text { Shahabuddin } \\
\text { et al. (2006) }\end{array}$ & $\begin{array}{l}\text { Threatened } \\
\text { status }\end{array}$ \\
\hline \multirow[t]{2}{*}{122} & Booted Eagle Hieraaetus pennatus & $A, F$ & 6 & 0 & - & 1 & LC \\
\hline & Falconidae & & & & & & \\
\hline 123 & Common Kestrel Falco tinnunculus & $\mathrm{F}$ & $3,4,5,6,7,9,10$ & C & 1 & 1 & LC \\
\hline 124 & Red-necked Falcon Falco chicquera & $\mathrm{F}$ & 7 & $\mathrm{~S}$ & - & - & LC \\
\hline 125 & Laggar Falcon Falco jugger & $\mathrm{F}$ & 6 & $\mathrm{~S}$ & - & 1 & NT \\
\hline 126 & Peregrine Falcon Falco peregrinus & $\mathrm{F}$ & 6 & $\mathrm{~S}$ & - & 1 & LC \\
\hline \multirow[t]{2}{*}{127} & Oriental Hobby Falco severus & - & - & - & 1 & - & LC \\
\hline & Podicipedidae & & & & & & \\
\hline 128 & Little Grebe Tachybaptus ruficollis & w & $1,2,8$ & A & 1 & 1 & LC \\
\hline \multirow[t]{2}{*}{129} & Great Crested Grebe Podiceps cristatus & W & 2 & $\mathrm{~S}$ & 1 & - & LC \\
\hline & Anhingidae & & & & & & \\
\hline \multirow[t]{2}{*}{130} & Darter Anhinga melanogaster & W & 1,8 & C & 1 & 1 & NT \\
\hline & Phalacrocoracidae & & & & & & \\
\hline 131 & Little Cormorant Phalacrocorax niger & W & $1,2,8$ & C & 1 & 1 & LC \\
\hline 132 & Indian Cormorant Phalacrocorax fuscicollis & W & 1,2 & C & 1 & 1 & LC \\
\hline \multirow[t]{2}{*}{133} & Great Cormorant Phalacrocorax carbo & W & 1,2 & C & 1 & 1 & LC \\
\hline & Ardeidae & & & & & & \\
\hline 134 & Little Egret Egretta garzetta & W & 2 & C & 1 & 1 & LC \\
\hline 135 & Grey Heron Ardea cinerea & - & - & - & 1 & - & LC \\
\hline 136 & Purple Heron Ardea purpurea & w & 1,2 & $u$ & 1 & - & LC \\
\hline 137 & Great Egret Casmerodius albus & w & 1,2 & $u$ & 1 & 1 & LC \\
\hline 138 & Intermediate Egret Mesophoyx intermedia & W & 2 & C & 1 & 1 & LC \\
\hline 139 & Cattle Egret Bubulcus ibis & $D, O, W$ & $1,2,3,4,5,8,10$ & C & 1 & 1 & LC \\
\hline 140 & Indian Pond Heron Ardeola grayii & w & $1,2,8$ & C & 1 & 1 & LC \\
\hline 141 & $\begin{array}{l}\text { Black-crowned Night Heron Nycticorax } \\
\text { nycticorax }\end{array}$ & W & 1,8 & $u$ & - & 1 & LC \\
\hline \multirow[t]{2}{*}{142} & Little Bittern Ixobrychus minutus & - & - & - & - & 1 & LC \\
\hline & Phoenicopteridae & & & & & & \\
\hline \multirow[t]{2}{*}{143} & Greater Flamingo Phoenicopterus ruber & - & - & - & 1 & - & LC \\
\hline & Threskiornithidae & & & & & & \\
\hline 144 & Glossy Ibis Plegadis falcinellus & W & 1,2 & $u$ & - & 1 & LC \\
\hline 145 & Black-headed Ibis Threskiornis melanocephalus & W & 2 & $\mathrm{~S}$ & 1 & 1 & LC \\
\hline 146 & Black Ibis Pseudibis papillosa & W & 2 & $u$ & 1 & - & LC \\
\hline \multirow[t]{2}{*}{147} & Eurasian Spoonbill Platalea leucorodia & W & 1,2 & C & 1 & 1 & LC \\
\hline & Pelecanidae & & & & & & \\
\hline 148 & Great White Pelican Pelecanus onocrotalus & W & 2 & $\mathrm{~S}$ & 1 & - & LC \\
\hline \multirow[t]{2}{*}{149} & Dalmatian Pelican Pelecanus crispus & & & & 1 & - & VU \\
\hline & Ciconiidae & & & & & & \\
\hline 150 & Painted Stork Mycteria leucocephala & W & 1,2 & $\mathrm{C}$ & 1 & 1 & NT \\
\hline
\end{tabular}




\begin{tabular}{|c|c|c|c|c|c|c|c|}
\hline & Family / Species & Habitat & *Locality & Abundance & $\begin{array}{c}\text { Sankar } \\
\text { et al. (1993) }\end{array}$ & $\begin{array}{l}\text { Shahabuddin } \\
\text { et al. (2006) }\end{array}$ & $\begin{array}{l}\text { Threatened } \\
\text { status }\end{array}$ \\
\hline 151 & Asian Openbill Anastomus oscitans & W & 1,2 & C & 1 & - & LC \\
\hline 152 & Black Stork Ciconia nigra & W & 2,4 & $u$ & 1 & 1 & LC \\
\hline \multirow[t]{2}{*}{153} & Woolly-necked Stork Ciconia episcopus & W & 1,2 & C & 1 & 1 & LC \\
\hline & Pittidae & & & & & & \\
\hline \multirow[t]{2}{*}{154} & Indian Pitta Pitta brachyura & $\mathrm{F}$ & 4,6 & $\mathrm{O}$ & 1 & 1 & LC \\
\hline & Laniidae & & & & & & \\
\hline 155 & Bay-backed Shrike Lanius vittatus & $\mathrm{D}, \mathrm{O}$ & 1,5 & 0 & 1 & - & LC \\
\hline 156 & Rufous-tailed Shrike Lanius isabellinus & $\mathrm{D}, \mathrm{F}, \mathrm{G}, \mathrm{O}$ & $1,2,3,4,5,6,7,8,9,10$ & c & - & 1 & LC \\
\hline 157 & Long-tailed Shrike Lanius schach & $D, F, G, O$ & $1,2,3,4,5,6,7,8,9,10$ & C & 1 & 1 & LC \\
\hline 158 & Great Grey Shrike Lanius excubitor & - & - & - & 1 & - & LC \\
\hline \multirow[t]{2}{*}{159} & Southern Grey Shrike Lanius meridionalis & $\mathrm{D}, \mathrm{F}, \mathrm{O}$ & $1,3,4,6,7,8,9$ & C & - & 1 & LC \\
\hline & Corvidae & & & & & & \\
\hline 160 & Rufous Treepie Dendrocitta vagabunda & $A, D, F, G, O$ & $1,2,3,4,5,6,7,8,9,10$ & C & 1 & 1 & LC \\
\hline 161 & House Crow Corvus splendens & $A, D, G, O$ & $1,2,3,4,5,6,7,8,9,10$ & C & 1 & - & LC \\
\hline 162 & Large-billed Crow Corvus macrorhynchos & $A, D, F, O$ & $1,2,3,4,5,6,7,9$ & C & 1 & 1 & LC \\
\hline 163 & Eurasian Golden Oriole Oriolus oriolus & $\mathrm{D}, \mathrm{F}, \mathrm{O}$ & $1,3,4,5,7$ & C & 1 & 1 & LC \\
\hline 164 & Large Cuckooshrike Coracina macei & $\mathrm{F}$ & $1,3,4,7,8,9,10$ & C & 1 & 1 & LC \\
\hline 165 & $\begin{array}{l}\text { Black-headed Cuckooshrike Coracina } \\
\text { melanoptera }\end{array}$ & - & - & - & 1 & 1 & LC \\
\hline 166 & Small Minivet Pericrocotus cinnamomeus & $\mathrm{F}, \mathrm{O}$ & $3,4,7,9$ & $u$ & 1 & 1 & LC \\
\hline 167 & $\begin{array}{l}\text { White-bellied Minivet Pericrocotus } \\
\text { erythropygius }\end{array}$ & $\mathrm{F}, \mathrm{O}$ & $1,4,6,7$ & $u$ & - & - & LC \\
\hline 168 & Scarlet Minivet Pericrocotus flammeus & - & - & - & 1 & - & LC \\
\hline 169 & White-browed Fantail Rhipidura aureola & $D, F, O$ & $1,3,6,7,10$ & $u$ & 1 & 1 & LC \\
\hline 170 & Black Drongo Dicrurus macrocercus & $A, D, F, G, O$ & $1,2,3,4,5,6,7,8,9,10$ & A & 1 & 1 & LC \\
\hline 171 & Ashy Drongo Dicrurus leucophaeus & $A, D, F, O$ & $1,3,4,6,7,9$ & C & - & - & LC \\
\hline 172 & White-bellied Drongo Dicrurus caerulescens & $\mathrm{D}, \mathrm{F}, \mathrm{O}$ & $3,4,6,7$ & $u$ & 1 & 1 & LC \\
\hline 173 & Asian Paradise-flycatcher Terpsiphone paradise & $\mathrm{F}, \mathrm{O}, \mathrm{W}$ & $1,3,4,5,7,9,10$ & $u$ & 1 & 1 & LC \\
\hline 174 & Common Iora Aegithina tiphia & - & - & - & 1 & - & LC \\
\hline 175 & Marshall's lora Aegithina nigrolutea & $\mathrm{F}$ & 3,6 & 0 & - & - & LC \\
\hline \multirow[t]{2}{*}{176} & $\begin{array}{l}\text { Common Woodshrike Tephrodornis } \\
\text { pondicerianus }\end{array}$ & $\mathrm{F}$ & $4,5,7,9$ & $u$ & 1 & 1 & LC \\
\hline & Muscicapidae & & & & & & \\
\hline 177 & Blue Rock Thrush Monticola solitarius & $D, F$ & 6,7 & $u$ & - & - & LC \\
\hline 178 & Orange-headed Thrush Zoothera citrina & $\mathrm{F}, \mathrm{O}$ & 7,9 & 0 & - & - & LC \\
\hline 179 & Tickell's Thrush Turdus unicolor & - & - & - & - & 1 & LC \\
\hline 180 & Grey-winged Blackbird Turdus boulboul & $\mathrm{F}$ & 4,7 & $\mathrm{~S}$ & - & - & LC \\
\hline 181 & Eurasian Blackbird Turdus merula & - & - & - & - & 1 & LC \\
\hline 182 & Dark-throated Thrush Turdus ruficollis & $\mathrm{F}$ & 6 & $\mathrm{~s}$ & - & - & LC \\
\hline 183 & Rusty-tailed Flycatcher Muscicapa ruficauda & - & - & - & - & 1 & LC \\
\hline
\end{tabular}




\begin{tabular}{|c|c|c|c|c|c|c|c|}
\hline & Family / Species & Habitat & *Locality & Abundance & $\begin{array}{c}\text { Sankar } \\
\text { et al. (1993) }\end{array}$ & $\begin{array}{l}\text { Shahabuddin } \\
\text { et al. (2006) }\end{array}$ & $\begin{array}{l}\text { Threatened } \\
\text { status }\end{array}$ \\
\hline 184 & Red-throated Flycatcher Ficedula parva & $\mathrm{D}, \mathrm{F}, \mathrm{O}$ & $1,3,4,6,7,9,10$ & C & 1 & 1 & LC \\
\hline 185 & Ultramarine Flycatcher Ficedula superciliaris & $\mathrm{F}$ & $3,6,7,9$ & $u$ & - & - & LC \\
\hline 186 & Verditer Flycatcher Eumyias thalassina & $\mathrm{F}$ & 6,7 & $\mathrm{~s}$ & - & 1 & LC \\
\hline 187 & Tickell's Blue Flycatcher Cyornis tickelliae & - & - & - & 1 & 1 & LC \\
\hline 188 & $\begin{array}{l}\text { Grey-headed Canary Flycatcher Culicicapa } \\
\text { ceylonensis }\end{array}$ & $\mathrm{D}, \mathrm{F}, \mathrm{O}$ & $1,4,9$ & 0 & 1 & 1 & LC \\
\hline 189 & Bluethroat Luscinia svecica & $\mathrm{O}, \mathrm{W}$ & $2,4,6,7,9,10$ & C & 1 & 1 & LC \\
\hline 190 & Oriental Magpie Robin Copsychus saularis & $A, D, F, O$ & $1,2,3,4,5,6,7,8,9,10$ & A & 1 & 1 & LC \\
\hline 191 & Indian Robin Saxicoloides fulicata & $A, D, F, G, O$ & $1,2,3,4,5,6,7,8,9,10$ & A & 1 & 1 & LC \\
\hline 192 & Black Redstart Phoenicurus ochruros & $D, F, G, O, W$ & $1,2,3,4,5,6,7,8,9,10$ & A & 1 & 1 & LC \\
\hline 193 & Blue-fronted Redstart Phoenicurus frontalis & $\mathrm{D}, \mathrm{O}, \mathrm{W}$ & $1,2,8$ & 0 & - & - & LC \\
\hline 194 & $\begin{array}{l}\text { White-capped Water Redstart Chaimarrornis } \\
\text { leucocephalus }\end{array}$ & W & 7 & S & - & - & LC \\
\hline 195 & Common Stonechat Saxicola torquata & $\mathrm{D}, \mathrm{F}, \mathrm{G}, \mathrm{O}$ & $1,3,4,5,6,9,10$ & C & 1 & 1 & LC \\
\hline 196 & Pied Bushchat Saxicola caprata & $D, G, O$ & $1,2,3,4,6,7,9,10$ & C & 1 & 1 & LC \\
\hline 197 & Grey Bushchat Saxicola ferrea & - & - & - & 1 & 1 & LC \\
\hline 198 & Desert Wheatear Oenanthe deserti & $D, G, O$ & $1,3,4,6,7,9,10$ & C & 1 & 1 & LC \\
\hline 199 & Isabelline Wheatear Oenanthe isabellina & - & - & - & - & 1 & LC \\
\hline \multirow[t]{2}{*}{200} & Brown Rock-chat Cercomela fusca & $D, F, G, O$ & $1,2,3,4,5,6,7,8,9,10$ & A & 1 & 1 & LC \\
\hline & Sturnidae & & & & & & \\
\hline 201 & Chestnut-tailed Starling Sturnus malabaricus & - & - & - & 1 & - & LC \\
\hline 202 & Brahminy Starling Sturnus pagodarum & $A, D, F, G, O$ & $1,2,3,4,5,6,7,8,9,10$ & A & 1 & 1 & LC \\
\hline 203 & Rosy Starling Sturnus roseus & $A, D, G, O$ & $1,3,4,5,6,7,9,10$ & C & 1 & 1 & LC \\
\hline 204 & Common Starling Sturnus vulgaris & $\mathrm{D}, \mathrm{F}, \mathrm{O}$ & $1,4,5,6,9,10$ & $u$ & 1 & 1 & LC \\
\hline 205 & Asian Pied Starling Sturnus contra & $D, F, G, O$ & $1,2,3,4,5,6,7,8,9,10$ & C & 1 & 1 & LC \\
\hline 206 & Common Myna Acridotheres tristis & $A, D, G, O$ & $1,2,3,4,5,6,7,8,9,10$ & C & 1 & 1 & LC \\
\hline \multirow[t]{2}{*}{207} & Bank Myna Acridotheres ginginianus & $A, D, O, W$ & $1,2,3,4,5,6,7,8,9,10$ & A & 1 & 1 & LC \\
\hline & Certhiidae & & & & & & \\
\hline \multirow[t]{2}{*}{208} & Spotted Creeper Salpornis spilonotus & - & - & - & 1 & - & LC \\
\hline & Paridae & & & & & & \\
\hline \multirow[t]{2}{*}{209} & Great Tit Parus major & $\mathrm{F}, \mathrm{W}$ & $1,2,4,7$ & $u$ & 1 & 1 & LC \\
\hline & Hirundinidae & & & & & & \\
\hline 210 & Plain Martin Riparia paludicola & - & - & - & 1 & - & LC \\
\hline 211 & Dusky Crag Martin Hirundo concolor & - & - & - & 1 & 1 & LC \\
\hline 212 & Barn Swallow Hirundo rustica & $\mathrm{A}, \mathrm{O}$ & $1,2,3,4,5,6,7,8,9,10$ & C & 1 & - & LC \\
\hline 213 & Wire-tailed Swallow Hirundo smithii & A & $1,2,3,4,5,6,7,8,9,10$ & C & 1 & 1 & LC \\
\hline \multirow[t]{2}{*}{214} & Red-rumped Swallow Hirundo daurica & A & $3,4,6,7,9,10$ & $\mathrm{u}$ & 1 & 1 & LC \\
\hline & Pycnonotidae & & & & & & \\
\hline 215 & Red-whiskered Bulbul Pycnonotus jocosus & $A, D, F, G$ & 2,5 & $\mathrm{~s}$ & - & - & LC \\
\hline
\end{tabular}




\begin{tabular}{|c|c|c|c|c|c|c|c|}
\hline & Family / Species & Habitat & *Locality & Abundance & $\begin{array}{c}\text { Sankar } \\
\text { et al. (1993) }\end{array}$ & $\begin{array}{l}\text { Shahabuddin } \\
\text { et al. (2006) }\end{array}$ & $\begin{array}{c}\text { Threatened } \\
\text { status }\end{array}$ \\
\hline 216 & White-eared Bulbul Pycnonotus leucotis & $A, D, F, G, O$ & $1,2,3,4,5,6,7,9,10$ & A & 1 & - & LC \\
\hline \multirow[t]{2}{*}{217} & Red-vented Bulbul Pycnonotus cafer & $A, D, F, G, O$ & $1,2,3,4,5,6,7,8,9,10$ & A & 1 & 1 & LC \\
\hline & Cisticolidae & & & & & & \\
\hline 218 & Zitting Cisticola Cisticola juncidis & $D, G, O$ & $2,4,9,10$ & C & - & - & LC \\
\hline 219 & Rufous-fronted Prinia Prinia buchanani & $D, F, G, O$ & $1,2,3,4,5,6,7,8,9,10$ & C & - & - & LC \\
\hline 220 & Grey-breasted Prinia Prinia hodgsonii & $D, F, G, O$ & $1,2,3,4,5,6,7,8,9,10$ & C & 1 & 1 & LC \\
\hline 221 & Jungle Prinia Prinia sylvatica & $D, F, G, O$ & $1,2,3,4,5,6,7,8,9,10$ & C & 1 & 1 & LC \\
\hline 222 & Ashy Prinia Prinia socialis & $D, F, G, O$ & $1,2,3,4,5,6,7,8,9,10$ & C & 1 & 1 & LC \\
\hline \multirow[t]{2}{*}{223} & Plain Prinia Prinia inornata & $D, F, G, O$ & $1,2,3,4,5,6,7,8,9,10$ & C & 1 & 1 & LC \\
\hline & Zosteropidae & & & & & & \\
\hline \multirow[t]{2}{*}{224} & Oriental White-eye Zosterops palpebrosus & $D, F$ & $1,2,3,4,5,6,7,9$ & C & 1 & 1 & LC \\
\hline & Sylviidae & & & & & & \\
\hline 225 & Cetti's Bush Warbler Cettia cetti & $D, F, O$ & $2,3,4,9$ & $u$ & - & - & LC \\
\hline 226 & $\begin{array}{l}\text { Moustached Warbler Acrocephalus } \\
\text { melanopogon }\end{array}$ & $\mathrm{O}, \mathrm{W}$ & 2,3 & U & - & - & LC \\
\hline 227 & $\begin{array}{l}\text { Clamorous Reed Warbler Acrocephalus } \\
\text { stentoreus }\end{array}$ & $D, G$ & $2,3,4,6,9,10$ & C & - & - & LC \\
\hline 228 & Booted Warbler Hippolais caligata & $D, G, F, O$ & $1,3,4,6,7,9,10$ & C & - & - & LC \\
\hline 229 & Common Tailorbird Orthotomus sutorius & $D, G, F, O$ & $1,2,3,4,5,6,7,9,10$ & A & 1 & 1 & LC \\
\hline 230 & Yellow-browed Warbler Phylloscopus inornatus & - & - & - & 1 & - & LC \\
\hline 231 & Common Chiffchaff Phylloscopus collybita & $\mathrm{F}, \mathrm{O}$ & $1,3,4,5,8,9,10$ & A & 1 & 1 & LC \\
\hline 232 & Plain Leaf Warbler Phylloscopus neglectus & $D, G, F, O$ & $1,2,3,4,5,6,7,8,9,10$ & C & - & - & LC \\
\hline 233 & Sulphur-bellied Warbler Phylloscopus griseolus & $\mathrm{G}, \mathrm{F}, \mathrm{O}$ & $1,4,5,6,7,8,10$ & C & - & 1 & LC \\
\hline 234 & Hume's Warbler Phylloscopus humei & $\mathrm{G}, \mathrm{F}, \mathrm{O}$ & $1,3,4,6,7,9$ & C & - & 1 & LC \\
\hline 235 & Greenish Warbler Phylloscopus trochiloides & $D, G, F, O$ & $3,4,6,7,9$ & C & 1 & 1 & LC \\
\hline 236 & $\begin{array}{l}\text { Large-billed Leaf Warbler Phylloscopus } \\
\text { magnirostris }\end{array}$ & $D, G, F, O$ & $1,2,3,4,6,7,9$ & C & - & - & LC \\
\hline 237 & $\begin{array}{l}\text { Western Crowned Warbler Phylloscopus } \\
\text { occipitalis }\end{array}$ & $\mathrm{G}, \mathrm{F}, \mathrm{O}$ & $1,3,6,7,9$ & C & - & - & LC \\
\hline 238 & Tawny-bellied Babbler Dumetia hyperythra & - & - & - & 1 & - & LC \\
\hline 239 & Yellow-eyed Babbler Chrysomma sinense & $D, G, F, O$ & $2,3,5,6,7,9,10$ & A & 1 & 1 & LC \\
\hline 240 & Common Babbler Turdoides caudatus & $D, G, F, O$ & $1,2,3,4,5,7,9,10$ & A & 1 & 1 & LC \\
\hline 241 & Large Grey Babbler Turdoides malcolmi & $D, G, F, O$ & $1,2,3,4,5,6,7,8,9,10$ & A & 1 & 1 & LC \\
\hline 242 & Jungle Babbler Turdoides striatus & $\mathrm{D}, \mathrm{F}, \mathrm{O}$ & $1,2,3,4,5,6,7,8,9,10$ & A & 1 & 1 & LC \\
\hline \multirow[t]{2}{*}{243} & Lesser Whitethroat Sylvia curruca & $D, G, F, O$ & $1,2,3,4,5,6,7,8,9,10$ & A & 1 & 1 & LC \\
\hline & Alaudidae & & & & & & \\
\hline 244 & Singing Bushlark Mirafra cantillans & - & - & - & 1 & - & LC \\
\hline 245 & Indian Bushlark Mirafra erythroptera & $G, F$, & $3,4,6,7,9,10$ & C & 1 & 1 & LC \\
\hline 246 & Ashy-crowned Sparrow Lark Eremopterix grisea & $\mathrm{G}, \mathrm{F}, \mathrm{O}$ & $3,5,6,8,9$ & C & - & 1 & LC \\
\hline 247 & $\begin{array}{l}\text { Greater Short-toed Lark Calandrella } \\
\text { brachydactyla }\end{array}$ & - & - & - & - & 1 & LC \\
\hline
\end{tabular}




\begin{tabular}{|c|c|c|c|c|c|c|c|}
\hline & Family / Species & Habitat & *Locality & Abundance & $\begin{array}{l}\text { Sankar } \\
\text { et al. (1993) }\end{array}$ & $\begin{array}{l}\text { Shahabuddin } \\
\text { et al. (2006) }\end{array}$ & $\begin{array}{l}\text { Threatened } \\
\text { status }\end{array}$ \\
\hline 248 & Rufous-tailed Lark Ammomanes phoenicurus & $\mathrm{G}, \mathrm{O}$ & $1,3,4,6,9,10$ & $u$ & - & 1 & LC \\
\hline 249 & Crested Lark Galerida cristata & $D, G, O$ & $1,2,5,9,10$ & C & - & 1 & LC \\
\hline \multirow[t]{2}{*}{250} & Oriental Skylark Alauda gulgula & $\mathrm{G}, \mathrm{O}$ & $3,6,7$ & $u$ & - & - & LC \\
\hline & Nectariniidae & & & & & & \\
\hline \multirow[t]{2}{*}{251} & Purple Sunbird Nectarinia asiatica & $A, D, F, O$ & $1,2,3,4,5,6,7,8,9,10$ & A & 1 & 1 & LC \\
\hline & Passeridae & & & & & & \\
\hline 252 & House Sparrow Passer domesticus & $\mathrm{D}, \mathrm{O}$ & $1,2,5,7,10$ & C & 1 & 1 & LC \\
\hline 253 & Spanish Sparrow Passer hispaniolensis & $\mathrm{D}, \mathrm{O}$ & $4,5,7$ & C & 1 & - & LC \\
\hline 254 & Eurasian Tree Sparrow Passer montanus & $\mathrm{D}, \mathrm{F}, \mathrm{G}, \mathrm{O}$ & $1,3,4,5,7,9,10$ & C & 1 & 1 & LC \\
\hline 255 & $\begin{array}{l}\text { Chestnut-shouldered Petronia Petronia } \\
\text { xanthocollis }\end{array}$ & $D, G$ & $3,5,6,7,9$ & $u$ & 1 & 1 & LC \\
\hline 256 & Forest Wagtail Dendronanthus indicus & W & 2,8 & U & - & - & LC \\
\hline 257 & White Wagtail Motacilla alba & W & $2,6,8$ & $u$ & 1 & 1 & LC \\
\hline 258 & $\begin{array}{l}\text { White-browed Wagtail Motacilla } \\
\text { maderaspatensis }\end{array}$ & W & 2,8 & C & 1 & 1 & LC \\
\hline 259 & Citrine Wagtail Motacilla citreola & W & $2,6,8$ & C & - & 1 & LC \\
\hline 260 & Yellow Wagtail Motacilla flava & W & 2,8 & U & 1 & - & LC \\
\hline 261 & Grey Wagtail Motacilla cinerea & W & $2,6,8$ & $u$ & 1 & 1 & LC \\
\hline 262 & Paddyfield Pipit Anthus rufulus & 0 & $2,5,7,8,9,10$ & C & 1 & - & LC \\
\hline 263 & Tawny Pipit Anthus campestris & $\mathrm{G}, \mathrm{O}$ & $1,2,4,8,9,10$ & C & - & - & LC \\
\hline 264 & Tree Pipit Anthus trivialis & $\mathrm{G}, \mathrm{O}$ & $1,2,5,8,9,10$ & C & 1 & 1 & LC \\
\hline 265 & Baya Weaver Ploceus philippinus & G & $1,2,5,9,10$ & C & 1 & 1 & LC \\
\hline 266 & Red Avadavat Amandava amandava & G & $1,2,3,4,6,7,8,9$ & C & 1 & - & LC \\
\hline 267 & Indian Silverbill Lonchura malabarica & $A, D, F, G, O$ & $1,2,3,4,5,6,7,8,9,10$ & A & 1 & 1 & LC \\
\hline \multirow[t]{2}{*}{268} & Scaly-breasted Munia Lonchura punctulata & G & $1,3,4,6,7,9,10$ & C & - & 1 & LC \\
\hline & Fringillidae & & & & & & \\
\hline 269 & Common Rosefinch Carpodacus erythrinus & $\mathrm{F}$ & $3,4,6,7,8,9$ & $u$ & 1 & 1 & LC \\
\hline 270 & Crested Bunting Melophus Iathani & - & - & - & 1 & - & LC \\
\hline 271 & Grey-necked Bunting Emberiza buchanani & - & - & - & 1 & - & LC \\
\hline 272 & Red-headed Bunting Emberiza bruniceps & - & - & - & - & 1 & LC \\
\hline 273 & White-capped Bunting Emberiza stewartii & $\mathrm{F}$ & $1,6,7,9$ & $\mathrm{U}$ & 1 & 1 & LC \\
\hline
\end{tabular}

Habitat: A - Aerial, D - Disturbed, F - Forested habitat, G - Grass dominant habitat, O - Open or scrub habitat, W - Water bodies and riparian habitat Locality: * see surveyed locality names in Figure 1

Abundance: A - Abundant, C - Common, O - Occasional, S - Stray, U - Uncommon

1 = present, $-=$ absent

Threatened status: CR - Critically Endangered, EN - Endangered, VU - Vulnerable, NT - Near Threatened, LC - Least Concern 